\title{
MRI and CSF oligoclonal bands after autologous hematopoietic stem cell transplantation in MS
}

\author{
A. Saiz, MD; E. Carreras, MD; J. Berenguer, MD; J. Yagüe, MD; C. Martínez, MD; P. Marín, MD; \\ M. Rovira, MD; T. Pujol, MD; T. Arbizu, MD; and F. Graus, MD
}

Article abstract-Objective: To analyze the MRI and CSF oligoclonal bands (OB) changes in patients with MS who underwent an autologous hematopoietic stem cell transplantation (AHSCT). Background: AHSCT is evaluated as an alternative therapy in severe MS. In previous series of AHSCT for MS, data on MRI or OB outcome were limited or not provided. Methods: Five patients with a median Kurtzke's EDSS score of 6.5, more than two attacks, and confirmed worsening of the EDSS in the previous year received an AHSCT. Hematopoietic stem cells were mobilized with cyclophosphamide $(3 \mathrm{~g} / \mathrm{m} 2)$ and granulocyte colony-stimulating factor $(5 \mu \mathrm{g} / \mathrm{kg} / \mathrm{d})$. The graft was $\mathrm{T}$ cell depleted by positive CD $34+$ selection. Conditioning regimen included BCNU $\left(300 \mathrm{mg} / \mathrm{m}^{2}\right)$, cyclophosphamide $(150 \mathrm{mg} / \mathrm{kg}$ in 3 days), and antithymocyte globulin (60 mg/kg in 4 days). MRI scans were scheduled at baseline and 1, 3, 6, and 12 months and OB analysis at baseline and 3 and 12 months post-AHSCT. Results: Four patients had a stable or improved EDSS after a median follow-up of 18 months (range, 12 to 24 months). The fifth patient's condition deteriorated during AHSCT. She partially improved and remained stable after month 3 after AHSCT. The baseline CSF OB persisted 1 year after AHSCT. MRI studies after AHSCT showed no enhanced T1 lesions and no new or enlarging T2 lesions. The median percentage change of T2 lesion load was $-11.8 \%$ (range, -26.6 to $-4.0 \%$ ). All patients had a decrease of corpus callosum area at 1 year (median, 12.4\%; range, $7.8 \%$ to $20.5 \%$ ) that did not progress in the two patients evaluated at 2 years after AHSCT. Conclusions: Although the persistence of CSF OB suggests the lymphocytes were not eliminated from the CNS, the follow-up MRI studies showed no enhanced T1 brain lesions and a reduction in the T2 lesion load that correlated with the clinical stabilization of MS after AHSCT.

NEUROLOGY 2001;56:1084-1089

Immune ablation with autologous hematopoietic stem cell transplantation (AHSCT) is evaluated as a potential treatment for severe cases of MS and other systemic autoimmune diseases. ${ }^{1,2}$ The rationale for using AHSCT to treat MS is based on isolated MS case reports of patients who underwent this treatment for a concomitant hematologic malignancy and the positive effect of syngeneic or autologous bone marrow transplantation on the prevention or remission of experimental allergic encephalomyelitis. ${ }^{3}$ The aim of AHSCT is to produce a profound T-cell depletion and to reconstitute an immune system with a new immune tolerance. Although this objective would be better accomplished by use of an allogeneic hematopoietic stem cell transplantation, the morbidity and mortality of this type of transplantation prevents its use in patients with MS.

Few studies of AHSCT in MS have been published. ${ }^{4-7}$ They addressed the feasibility of the procedure, but no detailed information was provided on the evolution of the MRI lesions or the CSF abnormalities that would help in the understanding of the effects of the AHSCT. We describe the clinical, MRI, and CSF evolution of the first five patients with MS receiving an AHSCT at our institution, after a median follow-up of 18 months.

Methods. Patients. The patients reported are the first five included in a prospective protocol to evaluate the safety of T cell-depleted AHSCT for patients with severe MS. The treatment protocol is described in the Appendix. Eligibility criteria include: 1) age between 18 and 60 years; 2) clinically definite secondary progressive (SPMS) or relapsing remitting (RRMS) with a Kurtzke's Expanded Disability Status Scale (EDSS) of 4.0 to $6.5 ; 3$ ) an increase in the EDSS by 1.0 point with an EDSS of 5.5 or less or 0.5 with an EDSS $>5.5$ over the previous year in spite of treatment with interferon or other immunotherapies that are stopped at least 1 month before the AHSCT. Patients with RRMS must have at least two relapses in the last year. Patients are excluded if there is significant history of medical illness precluding transplantation, cognitive deterioration, or severe atrophy seen in the brain MRI. The protocol is approved and monitored by the ethical and re-

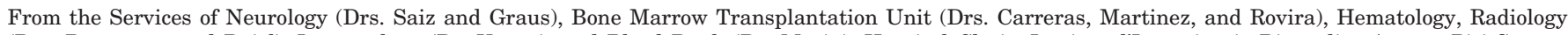

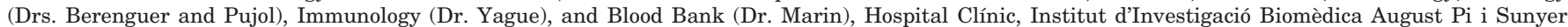
(IDIBAPS), University of Barcelona; and Service of Neurology (Dr. Arbizu), Unidad de Esclerosis Múltiple, C.S.U. de Bellvitge, L’Hospitalet, Spain. Supported by grant 97/001 Fundació La Marató TV3.

Received July 25, 2000. Accepted in final form December 30, 2000.

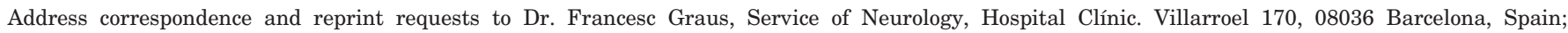
e-mail:graus@medicina.ub.es

1084 Copyright $\odot 2001$ by AAN Enterprises, Inc. 
search committees of the institution and the Spanish Ministry of Health.

Clinical evaluation. The neurologic status of the patients was evaluated (neurologic examination, EDSS, Ambulatory Index $[\mathrm{AI}]$ score) at baseline, at the end of stem cell mobilization, at months 1, 3, 6, 9, and 12 after AHSCT, and then every 6 months. Hematologic follow-up after hospital discharge and schedule of immunizations after treatment were done according to guidelines previously reported. ${ }^{8}$ Systemic adverse effects were scored by using the Bearman's scale. ${ }^{9}$

MRI evaluation. Brain MRI scans were obtained before the stem cell mobilization (baseline) and months 1, 3, 6, and 12 after AHSCT with a 1.5-T unit Siemens Magnetom SP (Erlangen, Germany) with a quadrature head coil. Two-dimensional gradient-echo scout images, acquired in three planes, were used for positioning slices in the MRI procedure. Sections with a thickness of $5 \mathrm{~mm}$ were acquired in the axial plane (parallel to the body of the corpus callosum) by using the following parameters: minimal interslice gap $(<1 \mathrm{~mm})$ and $192 \times 256$ matrix.

The MRI protocol consisted of sagittal spin-echo T1weighted images (repetition time [TR], $608 \mathrm{msec}$; echo time [TE], $14 \mathrm{msec}$ ), axial spin-echo T1-weighted images (TR/TE $608 / 14$ ), and sagittal fast spin-echo T2-weighted images (TR/TE 4600/90). Axial spin-echo T2-weighted images included proton density (TR/TE 2500/15) and T2 (TR/TE 2500/90). All T1-weighted images also were acquired 5 minutes after IV injection of $0.1 \mathrm{mmol} / \mathrm{kg}$ gadodiamide (Omniscan; Nycomed, Inc, Princeton, NJ).

A single experienced neuroradiologist (J.B.) visually identified and counted the number of hypointense and enhanced lesions in precontrast and postcontrast T1weighted images and hyperintense lesions in T2-weighted images. The measurement of lesion volume by using T2 sequences of the baseline and 1 year post-AHSCT MRI was calculated with a workstation Sienet DRC 104 (Siemens, Erlangen, Germany). Lesion volume calculations were obtained by using manual tracking, based on the lesional volume obtained by multiplying the total hyperintense lesion area by the thickness of the section and summation of the value of each section. The atrophy measures were based on the precontrast axial (lateral ventricle and brain widths) and sagittal (corpus callosum area) T1-weighted images and calculated in the workstation monitor. ${ }^{10}$ The mean coefficients of variation, obtained as described, ${ }^{10}$ were $1.5 \%, 0.3 \%$, and $5 \%$ for ventricle width, brain width, and corpus callosum area. All studies were done blind to the clinical evolution of the patients.

CSF analysis. Paired serum/CSF samples were obtained at baseline and 3 and 12 months after AHSCT and stored at $-80{ }^{\circ} \mathrm{C}$. Integrity of the blood-brain barrier (BBB) was estimated by the albumin index (upper normal value, 9.0). The identification of IgG-specific oligoclonal bands (OB) in CSF and serum was performed with an IgG-IEF Kit (Helena BioScience, Sunderland, UK) according to manufacturer's instructions. Basically, paired serum/CSF samples were run in an agarose gel isoelectric focusing, $\mathrm{pH} 3-10$. The separated proteins were transferred to a nitrocellulose membrane, immunofixed with sheep anti-human IgG peroxidase conjugated, and developed with 3-amino-9 ethylcarbazole (Sigma, St. Louis, MO). The patterns were interpreted qualitatively by com- paring the presence or absence of oligoclonal bands in CSF or serum.

Hematologic and immune evaluation. Peripheral blood was examined at baseline and months 3,9 , and 12 after transplantation, and then every 6 months. T cells (CD3+), helper/ inducer $\mathrm{T}$ cells $(\mathrm{CD} 3+\mathrm{CD} 4+)$, naive $(\mathrm{CD} 4+\mathrm{CD} 45 \mathrm{RA}+)$ and memory (CD4+CD45RO) helper/inducer T cells, suppressor/ cytotoxic T cells (CD3+CD8+), and B cells (CD19+) were analyzed on a FACScan (Becton Dickinson Immunocytometry Systems [BDIS], San Jose, CA). ${ }^{11}$

Results. Baseline patient characteristics. The five patients had a mean disease duration of 9 years (range, 6 to14 years). Two patients had RRMS and three had SPMS. The median scores of EDSS was 6.5 (range, 5.0 to 6.5), and that of AI, 4 (range, 2 to 6). Both patients with RRMS had six relapses the previous year despite treatment with interferon $\beta$ and repeated boluses of IV methylprednisolone (table 1).

Toxicity. There were no major systemic complications (table 1). Patient 5 presented an exacerbation of her leg weakness while receiving granulocyte colony-stimulating factor (G-CSF), which returned to baseline in a few days. Patient 2 developed a severe paraparesis concomitant with high fever associated with the administration of antithymocyte globulin (ATG), and her EDSS score increased from 6.5 to 8.0. She slowly improved to an EDSS of 7.5 over the ensuing 3 months. Four patients had uncomplicated urinary tract infections during the first 3 months after hospital discharge. Menstruation did not return in the oldest woman.

Neurologic outcome. At the last evaluation (median follow-up, 18 months; range, 12 to 24 months), the EDSS improved in Patients 4 (from 6.5 to 5.0) and 5 (from 6.5 to 5.5), was unchanged in Patients 1 and 3 , and remained stable after month 3 after AHSCT in Patient 2, whose condition worsened during the transplantation. Patient 3 had two relapses and Patient 4 one after AHSCT. All the episodes were subjective sensory symptoms that lasted a few days and did not require treatment. The neurologic examination during the relapse did not disclose any new sign, but the distribution of the sensory symptoms suggested an involvement of the spinal cord. Fatigue (although not formally measured) and urinary symptoms (once urinary infections were controlled) improved along the follow-up period in the five patients. None of the patients needed additional immunotherapy after AHSCT.

MRI evaluation. The baseline MRI characteristics are shown in table 2. Enhanced T1 lesions were presented in Patients 1, 3, and 5 and disappeared in the MRI done 1 month after AHSCT. The follow-up MRI studies did not show enhanced T1 lesions and no new hypointense T1 lesions, except in one patient who had a new hypointense lesion that corresponded to an enhancing lesion at baseline MRI. No new or enlarging hyperintense lesions on T2weighted images were observed at the follow-up MRI studies in the five patients. The median percentage change of T2 lesion volume was $-11.8 \%$ (range, -26.6 to $-4.0 \%$ ) (table 2).

A decrease in the corpus callosum area was observed in the five patients at 1 year (median decrease, $12.4 \%$; range, $7.8 \%$ to $20.5 \%$ ). The atrophy was independently confirmed by another neuroradiologist, with an interobserver median 
Table 1 Baseline clinical characteristics, number of reinfused cells, hematologic recovery, and toxicity of the treatment

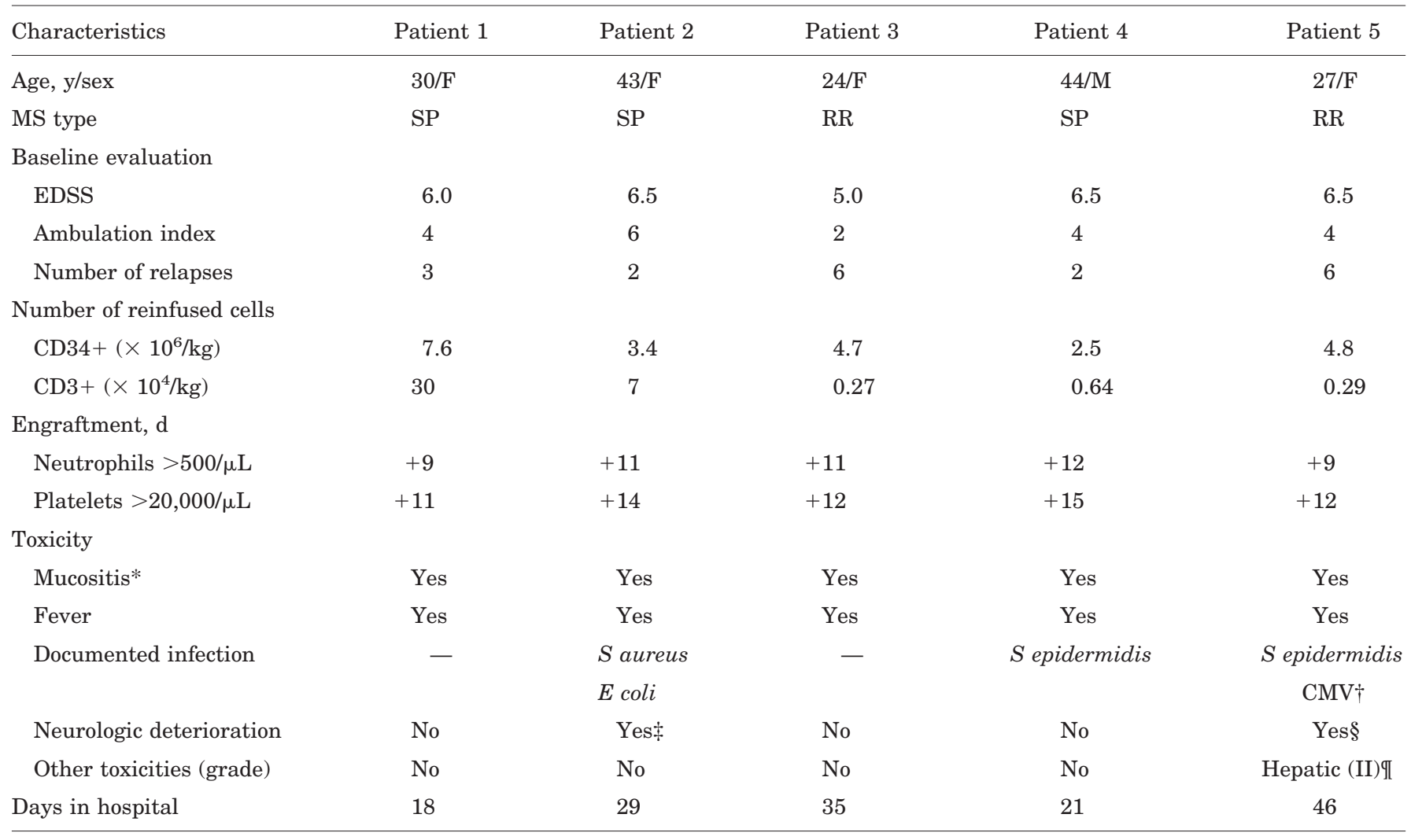

* Grade I = mucositis not requiring continuous IV analgesia.

$\dagger$ Asymptomatic reinfection.

$\ddagger$ During conditioning, incomplete recovery.

$\S$ During mobilization, complete recovery.

II Grade II $=$ AST/ALT $>5$ times of normal values.

$\mathrm{SP}=$ secondary progressive MS; RR = relapsing-remitting MS; EDSS = Expanded Disability Status Scale score.

variation in the percentage of the atrophy of the corpus callosum of $1.2 \%$ (range, $0.1 \%$ to $3.9 \%$ ). More than $50 \%$ of the reduction of the corpus callosum area occurred in the first 3 months after AHSCT in all of the patients. The percentage of loss during this period was $82 \%$ in Patient 2 and $80 \%$ in Patient 5 . The MRI 2 years after AHSCT done in Patients 1 and 2 showed no new enhanced or hypointense T1 lesions, a further reduction of the T2 lesion load, and the arrest in the decrease of the corpus callosum area seen during the first year after transplantation.

CSF evaluation. All patients showed OB in the baseline CSF examination. Patient 4 refused further lumbar punctures. At month 3 after AHSCT, Patients 1, 3, and 5 developed multiple $\mathrm{OB}$ in the serum, and many of them were also present in the CSF. Some of the observed OB in the baseline CSF were also identified in the serum. The intensity of these OB was stronger in the CSF than in the serum in Patients 1 and 5 (figure) and identical in Patient 3. The albumin index suggested a disruption of the BBB in the three patients (albumin index, 9.4, 14.8, and 20.1). Patient 2 did not present $\mathrm{OB}$ in the serum or disruption of the BBB at 3 months after AHSCT. At 1 year, the four patients (1, 2, 3, and 5) had the same CSF OB identified in the baseline CSF. In addition, some of the serum OB of Patients 1, 3, and 5, that appeared at month 3 persisted and were also identified in the CSF (figure).
Immune reconstitution after transplantation. All patients had a total number of peripheral blood lymphocytes $>500 / \mu \mathrm{L}$ and normal values of CD8 $+\mathrm{T}$ cells at 3 months after transplantation. CD4 $+\mathrm{T}$ cells achieved cell counts higher than $200 / \mu \mathrm{L}$ at 12 months after transplant in four of five patients. Throughout the period of the study, almost all CD4+ lymphocytes expressed CD45RO antigen (memory $\mathrm{T}$ cells), and only low numbers of cells expressed CD45RA antigen (naive $\mathrm{T}$ cells). All of the patients had normal values of B-lymphocytes at 9 months after treatment.

Discussion. AHSCT is an experimental treatment option for severe forms of MS. The efficacy of AHSCT remains to be demonstrated, and this treatment should be considered only in the setting of approved protocols. This study confirms previous work that patients with severe MS tolerate AHSCT with acceptable toxicity ${ }^{4-7}$ and shows for the first time three features that may be relevant for future studies. First, IgG OB in the CSF did not disappear after AHSCT; second, follow-up MRI showed a decrease in T2-weighted lesion load that persisted in absence of additional immunotherapy; and third, there was a decrease in corpus callosum area in spite of an ab- 
Table 2 MRI outcomes after autologous hematopoietic stem cell transplantation

\begin{tabular}{|c|c|c|c|c|c|}
\hline Outcomes & Patient 1 & Patient 2 & Patient 3 & Patient 4 & Patient 5 \\
\hline Mean T2 lesion volume, $\mathrm{cm}^{3}$ & 19.32 & 31.48 & 37.57 & 2.89 & 3.08 \\
\hline Enhanced T1 lesions & 12 & 0 & 5 & 0 & 6 \\
\hline Hypointense T1 lesions* & 22 & 18 & 22 & 5 & 2 \\
\hline \multicolumn{6}{|l|}{ Atrophy variables } \\
\hline Brain width, mm & 86 & 92 & 87 & 94 & 95 \\
\hline Corpus callosum area, $\mathrm{mm}^{2}$ & 685 & 523 & 461 & 594 & 515 \\
\hline \multicolumn{6}{|l|}{1 Year after transplantation } \\
\hline Percentage change of mean lesional volume from baseline & -19.7 & -26.6 & -10.3 & -4.0 & -11.8 \\
\hline Lateral ventricle width & 0 & 0 & +17.2 & +13.5 & 0 \\
\hline Brain width & -4.6 & 0 & -3.4 & 0 & 0 \\
\hline Corpus callosum area & -7.8 & -14.9 & -11.7 & -12.4 & -20.5 \\
\hline
\end{tabular}

* All lesions less than $1.3 \mathrm{~mm}$.

sence of inflammatory disease activity during the first year after AHSCT.

AHSCT was not associated with severe systemic toxicity. However, one of the patients presented a severe neurologic deterioration during the treatment that did not recover to the baseline level. Transient neurologic worsening is described in up to $42 \%$ of
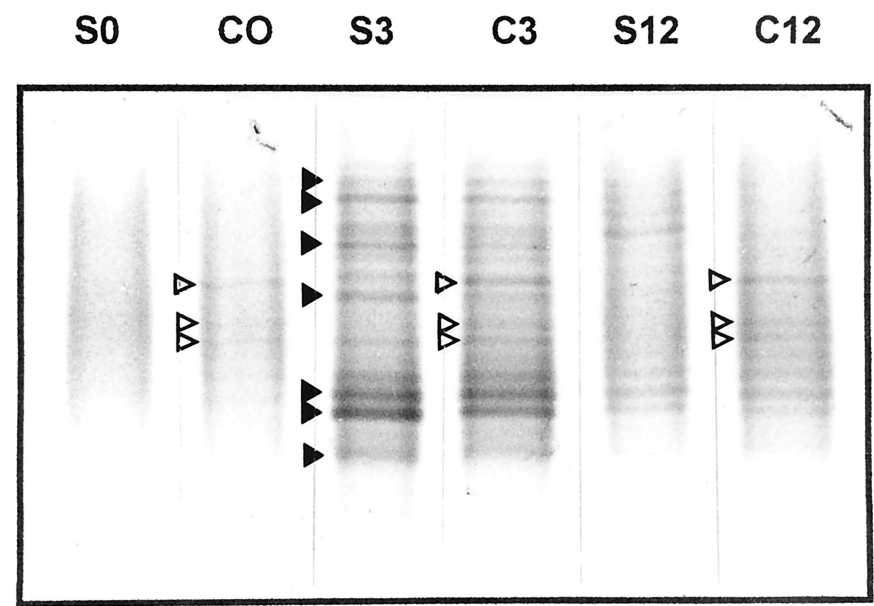

Figure. Identification of immunoglobulin G-specific oligoclonal bands (OB) from Patient 5. The baseline CSF had at least three $O B$ (open triangles). These bands persisted at month 3 after transplantation, and they were also seen in the serum but with a weaker intensity. At month 12, the three bands were only observed in the CSF. Serum at month 3 showed multiple $O B$ (closed triangles) that were also present in the CSF. Some of these bands were still present in the serum and CSF 12 months after treatment. $S O / C O=$ Baseline serum $/ C S F ; S 3 / C 3=$ serum $/ C S F 3$ months after transplantation; S12/C12 = serum/CSF 12 months after transplantation. patients during the procedure, ${ }^{7}$ but our patient emphasizes that this complication may be only partially reversible. Another patient had a transient neurologic deterioration while receiving granulocyte colony-stimulating factor (G-CSF) for stem cell mobilization. This patient had multiple relapses in the year previous to the transplantation and the possibility of a coincidence with the G-CSF treatment cannot be excluded. The mechanism of this complication is uncertain but has been reported, particularly when G-CSF alone is used to mobilize stem cell to the periphery. ${ }^{12}$ The current protocol uses half of the recommended dose of G-CSF ${ }^{1,2}$ with a priming dose of cyclophosphamide that may counteract possible immunostimulatory effects of G-CSF. We have not observed this complication in the subsequent six patients who have been treated with this protocol (unpublished).

The five patients' conditions remained neurologically stable or improved after discharge from the hospital. The absence of T1-enhancing lesions and the lack of new or enlarging hyperintense T2 lesions in the follow-up MRI suggests that AHSCT had a positive impact on active inflammation ${ }^{13}$ and seems to agree with the clinical stabilization of our patients in the first year after AHSCT. ${ }^{14}$ However, these MRI features are not a good predictor of long-term disability, and only the follow-up will unambiguously show whether the treatment has been able to modify the clinical course of these patients.

All patients showed an increase in the atrophy of the corpus callosum in spite of improvement of the other MRI variables. The relationship between inflammatory activity and the development of brain atrophy is unclear. In longitudinal studies of brain 
atrophy in untreated patients with RRMS, the decrease in corpus callosum area appears to be influenced by the baseline number of gadoliniumenhancing lesions. ${ }^{10}$ However, similar studies that used volumetric techniques failed to show a correlation of brain atrophy with enhanced lesion load. ${ }^{15}$ Taken these data together, one cannot rule out that the AHSCT could be effective in arresting the inflammatory activity but fail to reverse the pathologic process responsible for the brain atrophy. A second explanation is that the atrophy could be caused, at least in part, by the AHSCT. More than 50\% of the atrophy occurred in the 3 months after AHSCT, particularly in the two patients who had a more complicated AHSCT. This possibility would be further supported by the observation that the atrophy of the corpus callosum did not increase during the second year after the treatment. Brain atrophy was observed in patients undergoing allogeneic bone marrow transplantation for chronic myeloid leukemia, ${ }^{16}$ but specific measurements of the corpus callosum area were not provided. If the brain atrophy found in our patients is related to the treatment, this effect could be more evident in transplantation protocols that include total body radiation ${ }^{1,2}$ because of the potential synergistic effect of chemotherapy and radiation therapy in causing neurotoxicity. ${ }^{17}$

The CSF analysis 3 months after AHSCT showed a disruption of the $\mathrm{BBB}$ and the presence of many OB that were also identified in the serum. Serum OB was reported in up to $87 \%$ of patients who had an AHSCT for multiple myeloma and persisted 7 months on the average. ${ }^{18,19}$ The significance of these serum OB is unclear, but they could reflect recapitulation of early B-cell ontogeny. Persistence of CSF $\mathrm{OB}$ at 1 year after AHSCT was previously observed in two patients who were treated with busulfan, cyclophosphamide, and antithymocyte globulin. ${ }^{20}$ The serial analysis of the CSF OB at 3 and 12 months after AHSCT in our patients supports the idea that the B cells responsible for the IgG synthesis in the CNS survived the conditioning regimen. Unlike other CSF OB detected at 3 months after transplantation, at least some of the OB seen at the baseline CSF showed a stronger intensity in the CSF than in the serum. This observation suggests the B cells responsible for its synthesis were in the CNS, and the OB diffused to the serum because of the disruption of the BBB.

Unlike other published AHSCT protocols, ${ }^{4-7}$ we did not include in the conditioning regimen total body irradiation or added other drugs, as melphalan, etoposide, or cytosine arabinoside that in theory could be more effective to eradicate the lymphocytes in the CNS. We decided not to use these approaches for the concern of an increased incidence of cancer later in life. ${ }^{21-23}$ In addition, there is clinical ${ }^{24}$ and experimen$\mathrm{tal}^{25}$ evidence of neurologic deterioration after use of brain irradiation on demyelinating lesions.

The clinical impact of the presumable persistence of the lymphocytes in the CNS is unclear because the
MRIs did not show features compatible with disease activity, and the EDSS did not increase after hospital discharge. However, the evolution of CSF OB and the brain atrophy should be recorded in transplantation protocols to better understand the effects of $\mathrm{AH}$ SCT in MS.

\section{Appendix}

\section{Treatment protocol}

Mobilization of hematopoietic stem cells: Cyclophosphamide 3 $\mathrm{g} / \mathrm{m}^{2}+$ granulocyte colony-stimulating factor (G-CSF) $(5 \mu \mathrm{g} / \mathrm{kg} / \mathrm{d})$ Peripheral blood stem-cell collection: Fenwal CS3000 (Baxter) Leukapheresis: One T-cell-depleted + one non-T-cell-depleted (backup) (target in both: $3 \times 10^{6} \mathrm{CD} 34+/ \mathrm{kg}$ )

Positive CD34+ selection: Isolex 300 (Baxter) (Patients 1, 2)

CliniMACS (Miltényi) (Patients 3-5)

Conditioning regimen:

Day -6 -BCNU $(300 \mathrm{mg} / \mathrm{m} 2)$

Day -5-Antithymocyte globulin (Merieux) (ATG) (15 mg/kg), cyclophosphamide $(50 \mathrm{mg} / \mathrm{kg})$

Day -4-ATG $(15 \mathrm{mg} / \mathrm{kg})$, cyclophosphamide $(50 \mathrm{mg} / \mathrm{kg})$

Day -3-ATG (15 mg/kg), cyclophosphamide $(50 \mathrm{mg} / \mathrm{kg})$

Day -2-ATG $(15 \mathrm{mg} / \mathrm{kg})$

Day -1 -Rest

Day 0-Hematopoietic stem cell transplantation

Supportive care: Laminar airflow room; low microbial diet; oral ciprofloxacin, fluconazole, and acyclovir; inhaled pentamidine; IVIg, G-CSF (day +1), and ganciclovir if positive cytomegalovirus antigenemia

\section{Acknowledgment}

The authors thank Dr. B. Casanova, Hospital La Fe, Valencia, for referring Patient 4.

\section{References}

1. Tyndall A, Gratwohl A. Blood and marrow stem cell transplants in auto-immune disease: a consensus report written on behalf of the European League against Rheumatism (EULAR) and the European Group for Blood and Marrow Transplantation (EBMT). Bone Marrow Transplant 1997;19:643-645.

2. Comi G, Kappos L, Clanet M, et al. Guidelines for autologous blood and marrow stem cell transplantation in multiple sclerosis: a consensus report written on behalf of the European Group of Blood and Marrow Transplantation and the European Charcot Foundation. J Neurol 2000;247:376-382.

3. van Bekkum DW. New opportunities for the treatment of severe autoimmune diseases: Bone marrow transplantation. Clin Immunol Immunopathol 1998;89:1-10.

4. Burt RK, Traynor AE, Cohen B, et al. T cell-depleted autologous hematopoietic stem cell transplantation for multiple sclerosis: report of the first three patients. Bone Marrow Transplant 1998;21:537-541.

5. Kozàk T, Hauvdrovà E, Pit'ha J, et al. High-dose immunosuppressive therapy with PBPC support in the treatment of poor risk multiple sclerosis. Bone Marrow Transplant 2000;25:525531.

6. Burt RK, Traynor AE, Pope R, et al. Treatment of autoimmune disease by intense immunosuppressive conditioning and autologous hematopoietic stem cell transplantation. Blood 1998;92:3505-3514.

7. Fassas A, Anagnostopoulos A, Kazis A, et al. Autologous stem cell transplantation in progressive multiple sclerosis: An interim analysis of efficacy. J Clin Immunol 2000;20:24-30.

8. De la Cámara R, Bischofberger C, Campins R, et al. Inmunización postrasplante de progenitores hemopoyéticos: revisión y recomendaciones. Med Clin 1998;110:146-155.

9. Bearman SI, Appelbaum FR, Back A, et al. Regimen-related toxicity and early posttransplant survival in patients undergoing marrow transplantation for lymphoma. J Clin Oncol 1989; 7:1288-1294.

10. Simon JH, Jacobs LD, Campion MK, et al. A longitudinal study of brain atrophy in relapsing multiple sclerosis. Neurology 1999;53:139-148. 
11. Martinez C, Urbano-Ispizua A, Rozman C, et al. Immune reconstitution following allogeneic peripheral blood progenitor cell transplantation: comparison of recipients of positive CD34+ selected grafts with recipients of unmanipulated grafts. Exp Hematol 1999;27:561-568.

12. Openshaw H, Antel JP, Nash R, et al. Multiple sclerosis flares associated with recombinant granulocyte colony-stimulating factor. Neurology 2000;54:2147-2150.

13. Katz D, Taubenberger JK, Cannella B, et al. Correlation between magnetic resonance imaging findings and lesion development in chronic, active multiple sclerosis. Ann Neurol 1993; 34:661-669.

14. Molyneux PD, Filippi M, Barkhof F, et al. Correlations between monthly enhanced MRI lesion rate and changes in T2 lesion volume in multiple sclerosis. Ann Neurol 1998;43:332339.

15. Saindane AM, Ge Y, Udupa JK, et al. The effect of gadolinium-enhancing lesions on whole atrophy in relapsingremitting MS. Neurology 2000;55:61-65.

16. Jäger HR, Williams EJ, Savage DG, et al. Assessment of brain changes with registered MR before and after bone marrow transplantation for chronic myeloid leukemia. Am J Neuroradiol 1996;17:1275-1282.

17. DeAngelis LM, Shapiro WR. Drug/radiation interactions and central nervous system injury. In: Gutin PH, Leibel SA, Sheline GE, eds. Radiation injury to the nervous system. New York: Raven Press, 1991;361-382.

18. Mitus AJ, Stein R, Rappeport JM, et al. Monoclonal and oligoclonal gammopathy after bone marrow transplantation. Blood 1989;74:2764-2768
19. Zent CS, Wilson CS, Tricot G, et al. Oligoclonal protein bands and Ig isotype switching in multiple myeloma treated with high-dose therapy and hematopoietic cell transplantation. Blood 1998;91:3518-3523.

20. Openshaw H, Lund BT, Kashyap A, et al. Peripheral blood stem cell transplantation in multiple sclerosis with busulfan and cyclophosphamide conditioning: report of toxicity and immunological monitoring. Biol Blood Marrow Transpant 2000; 6:563-575.

21. Traweek ST, Slovak ML, Nademanee AP, et al. Myelodysplasia and acute myeloid leukemia occurring after autologous bone marrow transplantation for lymphoma. Leuk Lymphoma 1996;20:365-372.

22. Kollmannsberger C, Hartmann JT, Kanz L, et al. Risk of secondary myeloid leukemia and myelodysplastic syndrome following standard-dose chemotherapy or high-dose chemotherapy with stem cell support in patients with potentially curable malignancies. J Cancer Res Clin Oncol 1998;124:207214.

23. Curtis RE, Rowlings PA, Deeg J, et al. Solid cancers after bone marrow transplantation. N Engl J Med 1997;336:897904.

24. Peterson K, Rosenblum MK, Powers JM, et al. Effect of brain irradiation on demyelinating lesions. Neurology 1993;43: 2105-2112.

25. van Gelder M, van Bekkum DW. Effective treatment of relapsing experimental autoimmune encephalomyelitis with pseudoautologous bone marrow transplantation. Bone Marrow Transplant 1996;18:1029-1034.

\title{
The influence of cognitive impairment on driving performance in multiple sclerosis
}

\author{
Maria T. Schultheis, PhD; Edward Garay, BS; and John DeLuca, PhD
}

Article abstract-Objective: To examine the influence of impaired cognitive processing on measures of driving skills in persons with MS. Methods: Twenty-eight subjects with documented MS were divided into two groups-with [MS(+), n = 13] and without [MS(-), $\mathrm{n}=15]$ cognitive impairment-based on neuropsychological performance. Healthy control (HC) subjects $(n=17)$ matched on age and driving experience were also studied. Driving-related skills were compared between the groups based on performance on two computerized driving tests: the Useful Field of Vision (UFOV) and the Neurocognitive Driving Test (NDT). Results: The MS(+) group performed significantly worse than both the MS(-) and HC groups in the latency to perform several driving-specific functions on the NDT, but no overall group differences were observed in actual errors on the NDT. On the UFOV, when compared to $\mathrm{MS}(-)$ and $\mathrm{HC}$ subjects, the MS(+) group demonstrated poorer performance on two of the three subtests. Additionally, a significantly higher percentage of MS(+) individuals were rated within the high risk (probability of crash involvement) category, relative to the $\mathrm{MS}(-)$ and $\mathrm{HC}$ groups. Conclusions: Cognitive impairment can negatively affect driving-related skills in persons with MS and should be considered in the determination of driving ability.

NEUROLOGY 2001;56:1089-1094

The impact of cognitive impairment on driving skills and abilities has been documented in various neurologic populations, including brain injury, ${ }^{1-3}$ stroke, ${ }^{4,5}$ and dementia., ${ }^{6,7}$ These studies have identified de- creased attentional and visual perceptual skills, slowed information processing speed, and executive dysfunction as related to impaired driving skills and abilities. ${ }^{8-11}$ Despite recent evidence indicating the

From the Neuropsychology and Neuroscience Laboratory (Dr. Schultheis, E. Garay, and Dr. DeLuca), Kessler Medical Rehabilitation Research and Education Corporation, West Orange, NJ; and Departments of Physical Medicine and Rehabilitation (Drs. Schultheis and DeLuca) and Neuroscience (Dr. DeLuca), University of Medicine and Dentistry of New Jersey-New Jersey Medical School, Newark.

Supported by grant number PP0686 from the National Multiple Sclerosis Society.

Received July 19, 2000. Accepted in final form January 9, 2001.

Address correspondence and reprint requests to Dr. Maria T. Schultheis, Neuropsychology and Neuroscience Laboratory, Kessler Medical Rehabilitation Research \& Education Corporation, 1199 Pleasant Valley Way, West Orange, NJ 07052; e-mail: mschultheis@kmrrec.org 